\title{
Direct Dark Matter Search with XMASS
}

\author{
Shigetaka Moriyama*t \\ Kamioka Observatory, Institute for Cosmic Ray Research, The University of Tokyo \\ E-mail: moriyama@icrr.u-tokyo.ac.jp
}

\begin{abstract}
The XMASS project aims to search for dark matter, low-energy solar neutrino signals, and neutrinoless double-beta decay. The first prototype detector, XMASS-I, successfully provided sensitive searches of various types: low-mass weakly interacting massive particles, dark matter searches by means of annual modulation, double-electron capture, inelastic scattering on ${ }^{129} \mathrm{Xe}$, and bosonic superWIMPs. To increase the sensitivity, we are planning to construct larger detectors using improved photosensors. With the expected background of $10^{-5} \mathrm{~kg}^{-1} \mathrm{keV}^{-1} \mathrm{day}^{-1}$ caused by $p p$ solar neutrinos, a sensitivity to the WIMP-nucleon cross section smaller than $10^{-46} \mathrm{~cm}^{2}$ is expected. This enables us to search for WIMPs in the parameter region predicted by supersymmetric theories. It will also be highly sensitive to other candidates, such as bosonic superWIMPs giving traces as electron-recoil events.
\end{abstract}

The 11th International Workshop Dark Side of the Universe 2015

14-18 December 2015

Kyoto, Japan

\footnotetext{
* Speaker.

On behalf of the XMASS collaboration
} 


\section{Single-phase liquid xenon detector: XMASS project}

The XMASS project was proposed to use single-phase liquid-xenon detectors to observe rare events such as elastic scattering of nuclei by dark matter particles, neutrino-less double beta decay, and elastic scattering of electrons caused by $\mathrm{pp} /{ }^{7} \mathrm{Be}$ solar neutrinos. The advantages of liquid xenon are its large atomic number $(Z=54)$, high density $(3 \mathrm{~g} / \mathrm{cc})$, high light yield as a scintillator, absence of long-life radioisotopes, availability of purification methods, and scalability. As shown in Fig. 1 (left), the large atomic number and high density are useful for shielding against radiations coming outside the target volume and realizing a low background at the central part. On the contrary, dark matter candidates, such as weakly-interacting massive particles (WIMPs), uniformly interact over the sensitive volume, and a sensitive dark matter search can be conducted by extracting events happening deep inside the detector as shown in Fig. 1 (right). A high light yield is essential for realizing this approach, and it enables us to reconstruct event vertices based on, for example, information from photomultipliers (PMTs) surrounding the detector's target volume.

The availability of purification methods is important for reducing background sources such as krypton and radon in the liquid xenon target. Finally, the scalability is an important feature of the single-phase liquid xenon detector for future extensions to realize more sensitive detectors.
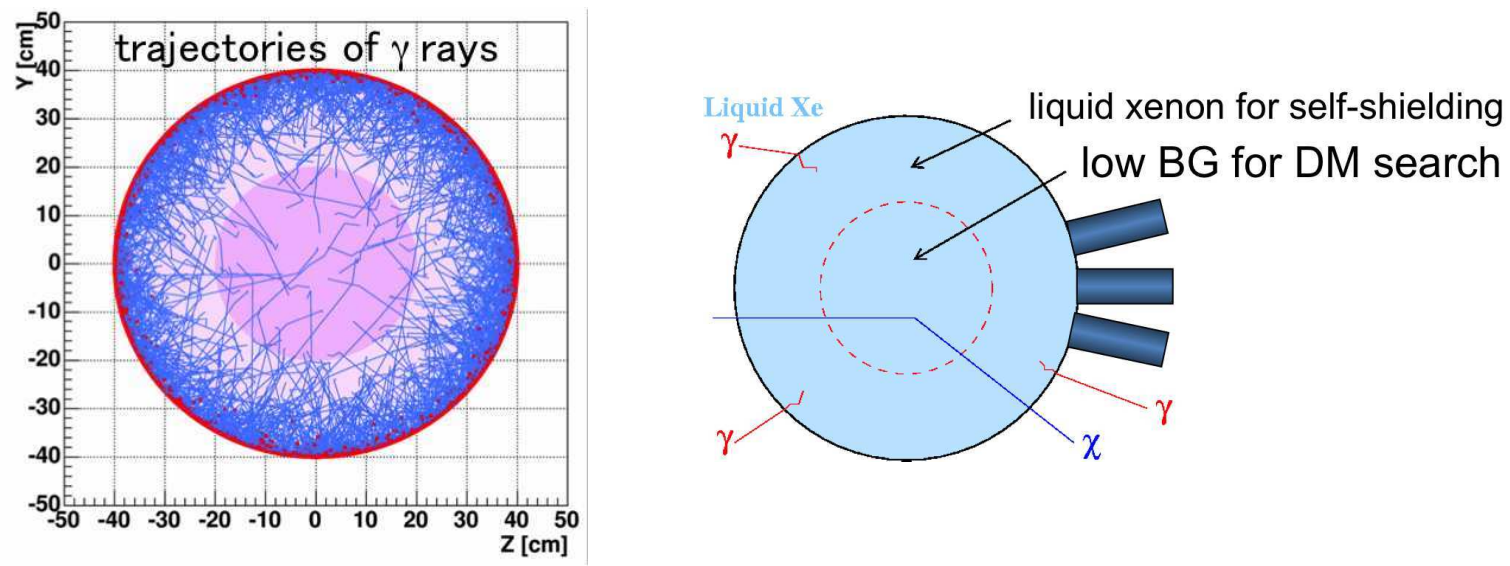

Figure 1: Self shielding of external radiation by liquid xenon itself (left). Because of its high atomic number $(Z=54)$ and density $(3 \mathrm{~g} / \mathrm{cc})$, strong absorption of radiation is expected. This causes a low background toward the center part of the sensitive volume. In contrast, dark matter particles uniformly interact over the sensitive volume (right). A sensitive search for dark matter can be conducted if the event vertex can be reconstructed based on information from the photomultiplier tubes. Three rectangular boxes show a part of the PMTs.

\section{XMASS-I detector}

As the first phase of the XMASS project, a detector with $835 \mathrm{~kg}$ of liquid xenon in its sensitive volume was constructed. The sensitive volume has a spherical shape with $\sim 80 \mathrm{~cm}$ diameter and is surrounded by 642 2-inch PMTs. Most of the PMTs have photocathodes (quantum efficiency $\sim 30 \%$ ) of hexagonal shape that cover more than $62 \%$ of the inner surface. Data acquisition is triggered if hits ( $>0.2$ p.e.) in four (ten) or more PMTs occur within a 200-ns timing window after 
the year 2013 (during the years 2010-2012). The waveform from each PMT is recorded by 10-bit $\operatorname{FADC}(1 \mathrm{Gs} / \mathrm{s})$.
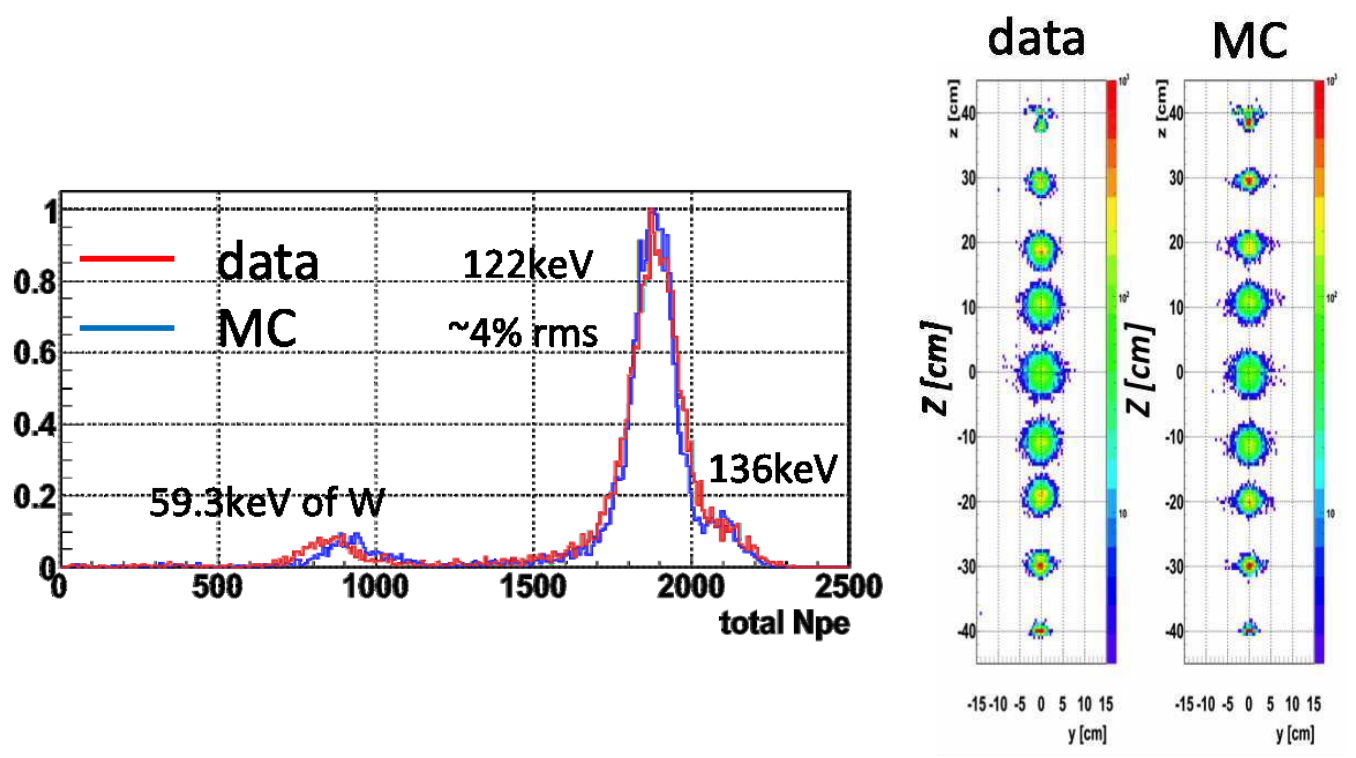

Figure 2: Distribution of the total number of photoelectrons with a ${ }^{57} \mathrm{Co}$-source at the center of the detector (left). Peaks are due to $122-\mathrm{keV}$ gamma rays, 136-keV gamma rays, and 60-keV fluorescence X-rays from a tungsten wire inside. Reconstructed vertex distribution at various positions inside the detector along its $Z$-axis (right). Real data are reproduced by Monte Carlo simulation.

By introducing a calibration source in the needle shape along the vertical axis of the sensitive volume, calibration data can be taken regularly. With a ${ }^{57} \mathrm{Co}$ source, almost 14 photoelectrons (p.e.s)/keV can be obtained at the center of the sensitive volume (Fig. 2, left). This high light yield is a prominent feature of the single-phase liquid xenon detector where the sensitive volume can be surrounded by PMTs. Figure 2 (right) shows the reconstructed vertex distribution of the data with the ${ }^{57} \mathrm{Co}$ thin source at various points in the detector.

The detector is located at the center of a cylindrical water tank that has a height of $11 \mathrm{~m}$ and diameter of $10 \mathrm{~m}$. It is useful as a passive shield and has 72 20-inch PMTs, such that it can be used as a muon veto as well. This is the first case of a dark matter experiment equipped with a water Cherenkov muon veto.

\section{Results from XMASS-I}

The XMASS-I detector has following features: the large photoelectron yield (14 p.e./keV), large mass, and low background. The following physics searches have been conducted based on these features. In particular, we achieved unprecedented sensitivity in the 40 to $120 \mathrm{keV}$ energy range by exploiting the low background at a level of $10^{-4} \mathrm{~kg}^{-1} \mathrm{keV}^{-1} \mathrm{day}^{-1}$ in the fiducial volume.

\subsection{Low-mass WIMPs}

The nature of dark matter is still unknown. Theories beyond the standard model predict some candidates for dark matter. Among the most plausible, the supersymmetric particles are the most 

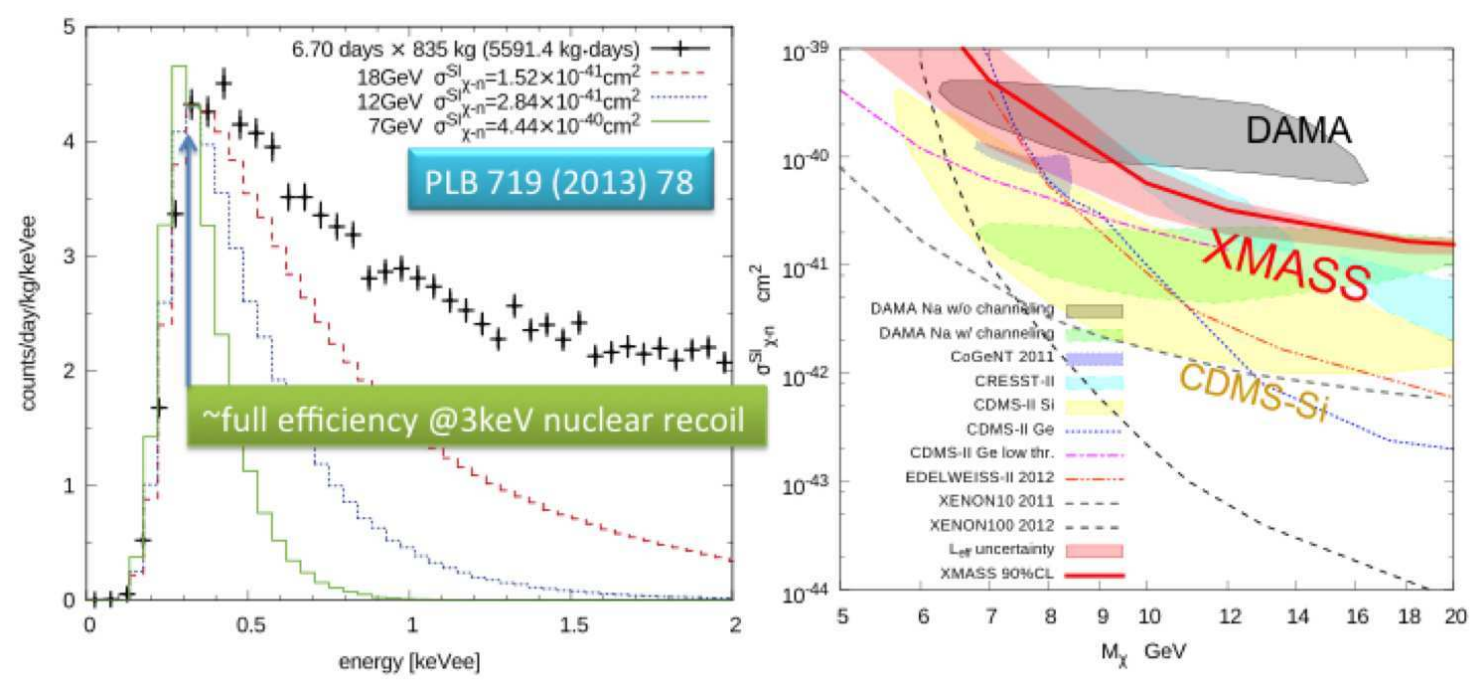

Figure 3: Simulated WIMP energy spectra in the XMASS detector assuming a maximum cross section that provides a signal rate no larger than the observation in any bin above 0.3 keVee where we have almost full efficiency (left). Limits and favored regions of the spin-independent elastic WIMP-nucleon cross section as a function of the WIMP mass (right) [1].

popular. In general, they are expected to have a mass of the order of $100 \mathrm{GeV}$; however, some experiments indicate a possible WIMP signal with a lighter mass of $10 \mathrm{GeV}$ and with a spinindependent cross section of the order of $10^{-40} \mathrm{~cm}^{2}$. The XMASS detector is sensitive to the low-energy deposition caused by these low mass WIMPs because of the high light yield of the detector. Figure 3 (left) shows the comparison between the observed energy spectrum $(5,591 \mathrm{~kg}$ day) and expected energy spectrum assuming the maximum cross section that provides a signal rate no longer than the observation in any bin above 0.3 keVee [1]. The right panel shows the limit of the cross section as a function of WIMP mass. XMASS excludes part of the parameter space favored by other experiments.

\subsection{Annual modulation}

A search for dark matter was conducted by looking for an annual modulation signal due to the Earth's rotation around the Sun. The data used for this analysis is taken from 359 live days $\times 832$ $\mathrm{kg}$ of exposure [2], which is comparable to the experiment that provided a positive signature of the modulation signal, as performed by the DAMA group [3].

Two types of analysis were conducted. One was a model-independent-analysis which made no assumptions about the energy spectra caused by dark matter particles. To search for any modulated signal in our observed data, an observed amplitude and limits for each energy bin were evaluated. Figure 4 shows the observed amplitude of annual modulation in each energy bin. No significant modulated signal was observed in the data. The maximum amplitude of $\sim 2.5 \times 10^{-2}$ events $/$ day $/ \mathrm{kg} / \mathrm{keVee}$ between 2.5 and $3.0 \mathrm{keVee}$ was obtained by DAMA while XMASS obtained an upper limit of $1.7 \times 10^{-2}$ events $/ \mathrm{day} / \mathrm{kg} / \mathrm{keVee}$. It is important to emphasize that the result also covered possible contributions caused by electron recoil that may explain DAMA's result because they did not discriminate between nuclear and electron recoil in their analysis. 


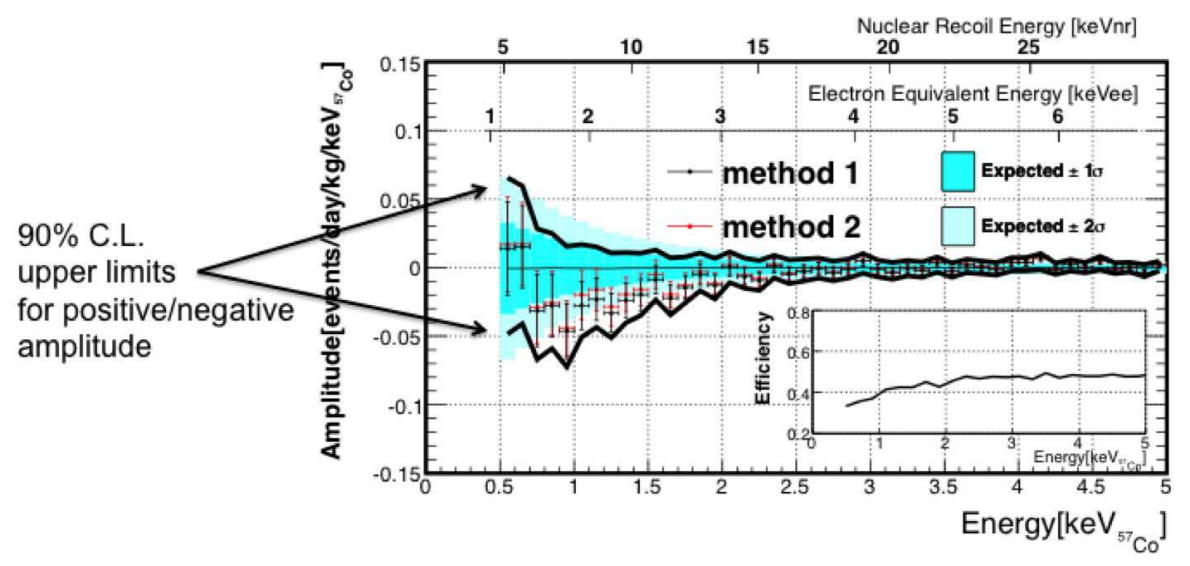

Figure 4: Modulation amplitude as a function of energy and limits for each energy bin [2]. Two independent methods were applied and gave consistent results. Solid lines represent $90 \%$ positive/negative upper limits on the amplitude. The colored bands show the expected amplitude in the case of no signal.

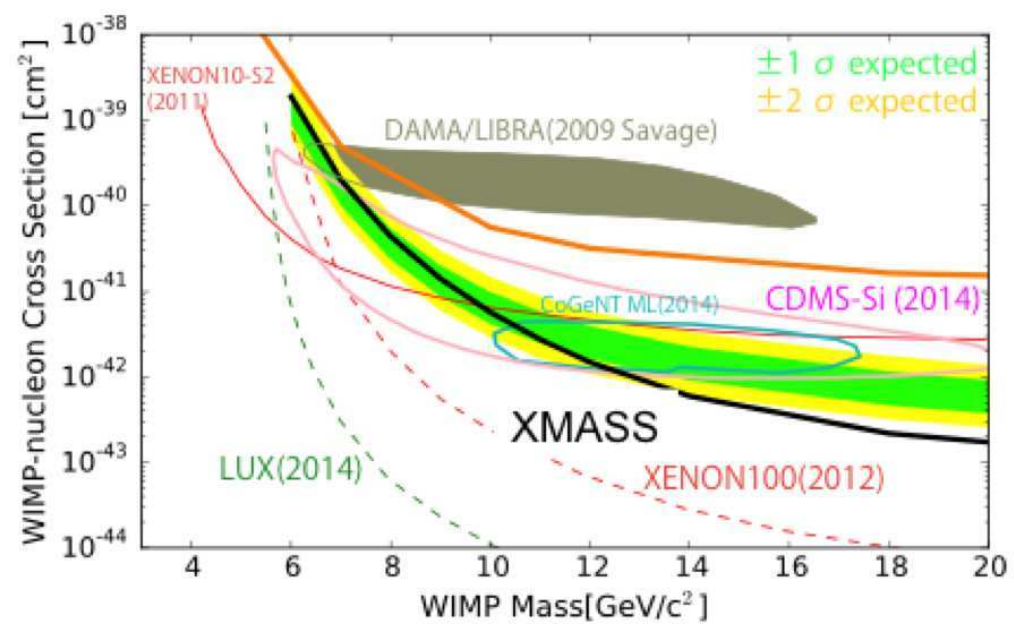

Figure 5: Limits on the spin-independent elastic WIMP-nucleon cross section as a function of WIMP mass [2].

The other analysis was based on the assumption that the modulation was caused by nuclear recoil from collisions with light-mass WIMPs. In this analysis, observed events were assumed to comprise of constant background plus a sinusoidal variation caused by WIMPs. The amplitude of the sinusoidal variation can be interpreted as the cross section of a WIMP to a nucleon. The energy dependence of the sinusoidal variation was evaluated by Monte Carlo simulation for WIMPs of various masses. To extract the amplitude from the data, event rates subdivided into two-dimensional bins (energy vs. observed period) were fitted by the function above. As a result, the fitted amplitudes for various masses of WIMPs were consistent with zero and the upper limit of the cross section is shown in Fig. 5. The region allowed by the DAMA experiment is almost excluded by our 
data. This is the first extensive search probing this region with an exposure comparable to theirs.

\subsection{Double-electron capture}

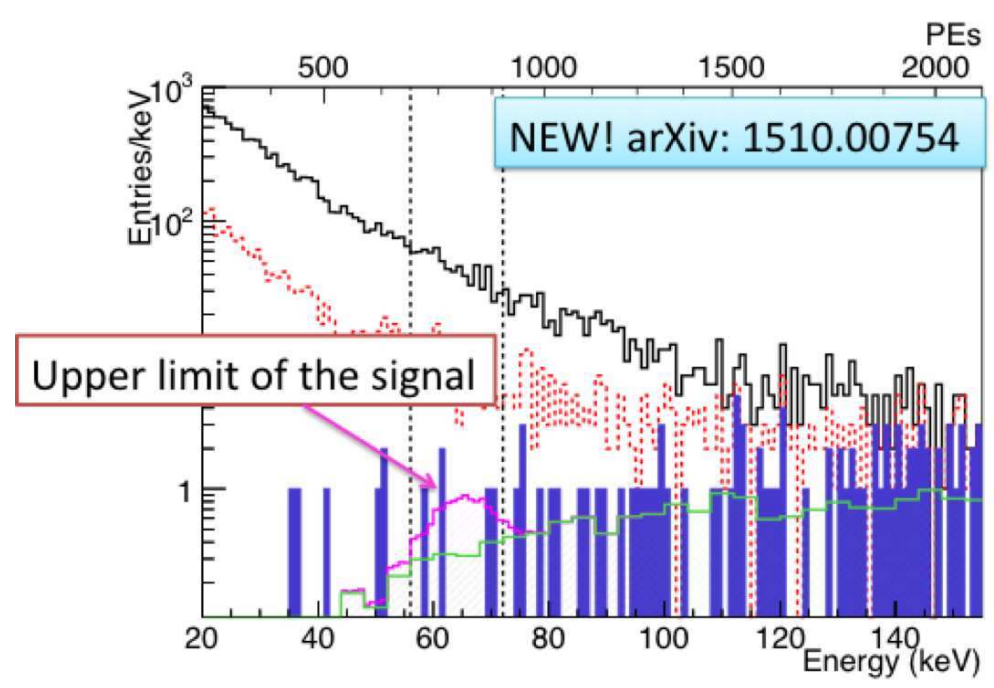

Figure 6: Observed energy spectrum and upper limit of the signal by the double-electron capture of ${ }^{124} \mathrm{Xe}$. The solid black line, red dashed line, and filled blue histograms show energy spectra for each step of the reduction. The green open histogram shows the expected background caused by ${ }^{214} \mathrm{~Pb}$ and the magenta open histogram shows the upper limit of the signal.

Double-electron capture (DEC) is a rare nuclear decay process wherein two orbital electrons are simultaneously captured. This process is allowed in the standard model of particle physics, but only a few positive experimental results exist so far. Any measurement of the half-life of the two-neutrino mode would provide a new reference for the calculation of nuclear matrix elements. In particular, ${ }^{124} \mathrm{Xe}$ is a candidate nucleus for providing information about the matrix element from the proton-rich side of the mass parabola of even-even isobars. The signal of ${ }^{124} \mathrm{Xe}$ DEC is expected to form a peak in an energy distribution at $63 \mathrm{keV}$, which is the sum of two K-shell X-ray energies. A search was performed using data collected over 165.9 days. Figure 6 shows the energy spectrum after each reduction step. The blue hatched histogram shows the final sample in the $15-\mathrm{cm}$ radius fiducial volume. The green open histogram shows the expected background due to ${ }^{214} \mathrm{~Pb}$. Its amount was determined by Bi-Po consecutive decay. As can be seen in the figure, no significant excess above the background was observed. The magenta open histogram shows the upper limit of the signal and the corresponding lower limit on the half-life is $4.7 \times 10^{21} \mathrm{yr}$ at a $90 \%$ confidence level. This stringent limit excludes some theoretical expectations. This demonstrates that the XMASS detector has the potential to provide useful information for nuclear physics.

\subsection{Inelastic scattering on ${ }^{129} \mathrm{Xe}$}

The experimental signature of WIMP detection through elastic scattering is a presence of featureless exponential rise towards the energy-threshold of a detector. This sometimes introduces 


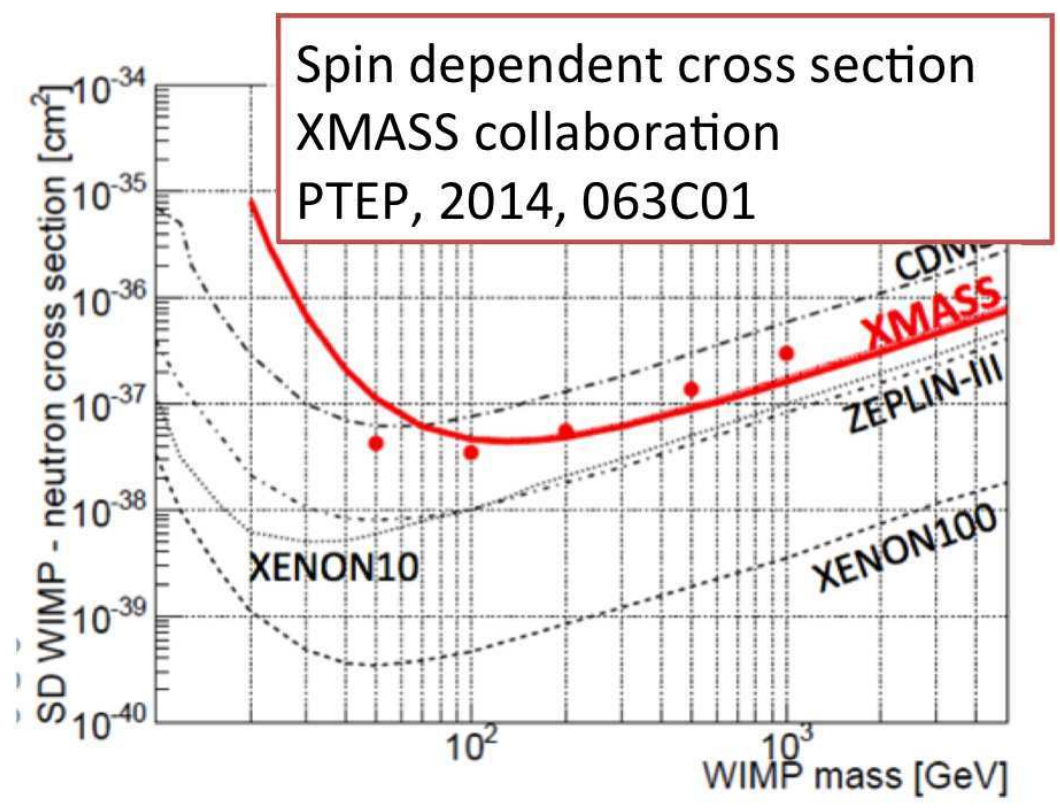

Figure 7: The thick red line and dots represents our limit [5] using different form factors for ${ }^{129}$ Xe. Our limit (90\% confidence level) is the first derived exclusively from data on inelastic scattering.

difficulty in distinguishing the signal from the background near the threshold. Assuming that a target nucleus has low-lying nuclear states, inelastic scattering on the nucleus would yield a readily identifiable signature; de-excitation after the inelastic scattering can cause a peak in an energy spectrum corresponding to the excitation energy of the nucleus.

Among the xenon isotopes, ${ }^{129} \mathrm{Xe}$ has a state at $39.58 \mathrm{keV}$ with a natural abundance of $26.4 \%$. The expected signal was estimated by Monte Carlo simulation including the energy deposition caused by associated nuclear recoil. We used the data set to search for the double-electron capture events and optimized the selection criteria for this particular search. As a result, no significant excess at the expected energy range was observed and an upper limit to the number of inelastic scattering events was derived. Figure 7 shows the corresponding upper limits to the spin-dependent WIMP-nucleon cross section. Since there are theoretical uncertainties in the form factor, which is necessary for calculating the WIMP-nucleus cross section from the WIMP-nucleon cross section, two independent form factors were used and the resultant upper limits are shown in Fig. 7. Our own limit is the first derived exclusively from data for inelastic scattering.

\subsection{Bosonic superWIMPs}

The cold dark matter (CDM) paradigm has been quite success at explaining the cosmological properties of the universe, such as the primordial cosmic microwave background and large-scale structure formation of the universe. However, simulations based on this CDM scenario expect richer structure on galactic scales than those observed. Lighter particles in the keV-to-MeV region may soften the problem and have been proposed in the literature. Among them, the bosonic super-weakly-interacting massive particles (superWIMPs) are good candidates and have not been experimentally constrained. Interestingly, these superWIMPs behave like massive photons and are 


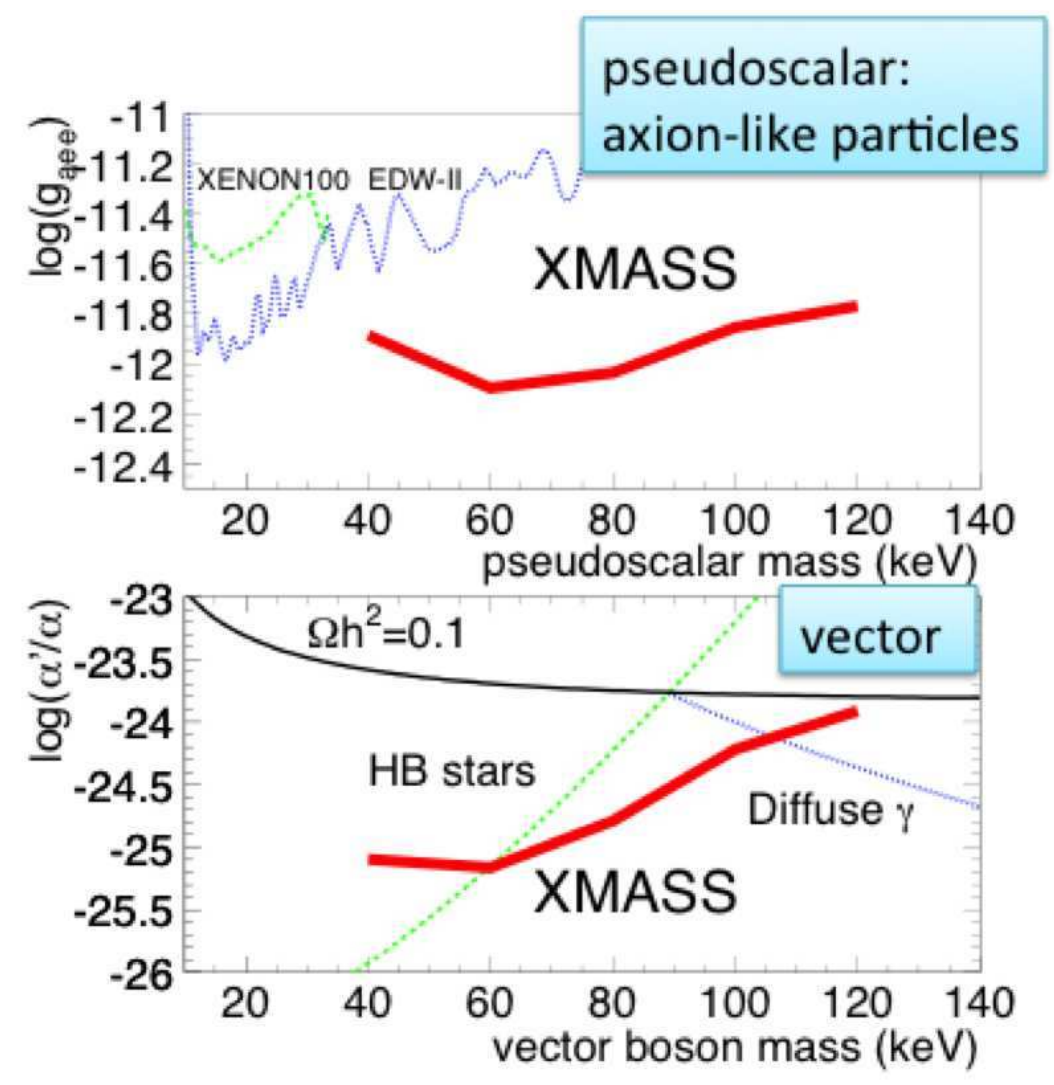

Figure 8: Limits on the coupling constant between electrons and pseudoscalar (axion-like) superWIMPs, $g_{\text {aee }}$ (top), and on the analog of the fine structure-constant for vector boson superWIMPs, $\alpha^{\prime}$ (bottom) [6]. The latter excludes $\alpha^{\prime}$, which explains dark matter density by thermal production in this mass range. This is the first experimental search for vector bosonic superWIMPs in this mass range.

absorbed by atoms, which then release energy equivalent to the superWIMPs's rest mass. Thus, we can expect a monochromatic peak that can be easily discriminated from the background.

The data-set for searching for the superWIMPs was the same as that used in the analyses above. Since the background below $40 \mathrm{keV}$ increases because of the background at the wall and theoretical calculation is limited up to $\sim 100 \mathrm{keV}$, superWIMPs with rest masses in the range of 40 to $120 \mathrm{keV}$ were searched for. We observed no signal but did obtain the constraint shown in Fig. 8. For the vector boson superWIMPs, the limit rejected the coupling to electrons that explains dark matter density by the thermal production of superWIMPs in this mass range.

\section{Future of XMASS}

The XMASS-I successfully provided sensitive searches for various types of dark matter. We are planning to construct a larger detector, XMASS-1.5, with 5 tons of liquid xenon and 1ton of fiducial mass. To achieve a higher sensitivity, we need to solve problems related to the background events happening at the inner surface of the XMASS-I detector. PMTs used in XMASS-I have flat photocathodes, as shown in Fig. 9 (left). In this case, scintillation photons from the events 


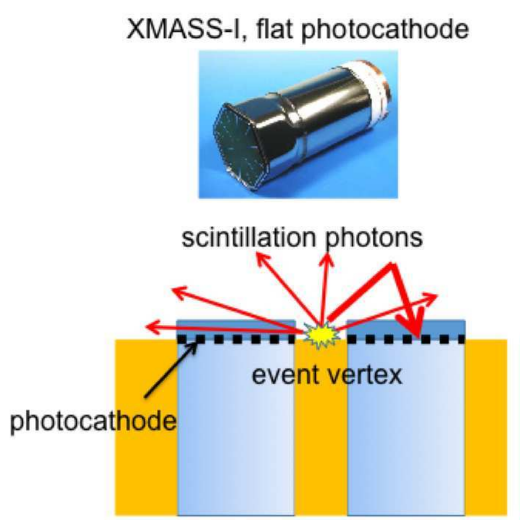

XMASS-1.5, dome-shape photocathode
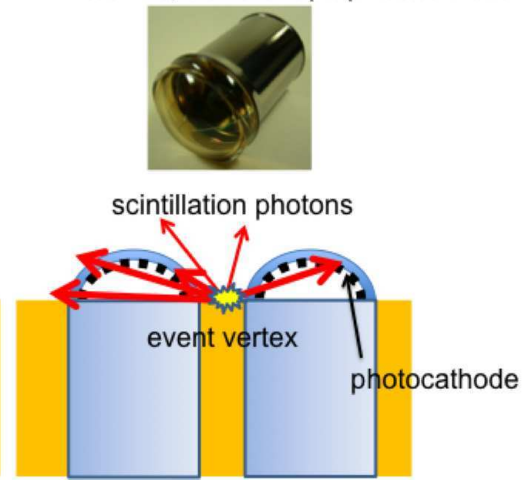

Figure 9: Advantage of PMTs with a photocathode of a convex shape. Scintillation lights originating on the inner wall of the detector were not efficiently detected by a flat photocathode used in XMASS-I (left). This caused an increase of the background inside a fiducial volume due to misconstruction. On the contrary, PMTs with a photocathode of a convex shape detected direct lights from the event vertex in an efficient way. This enabled us to reduce misconstruction down to a negligible level.
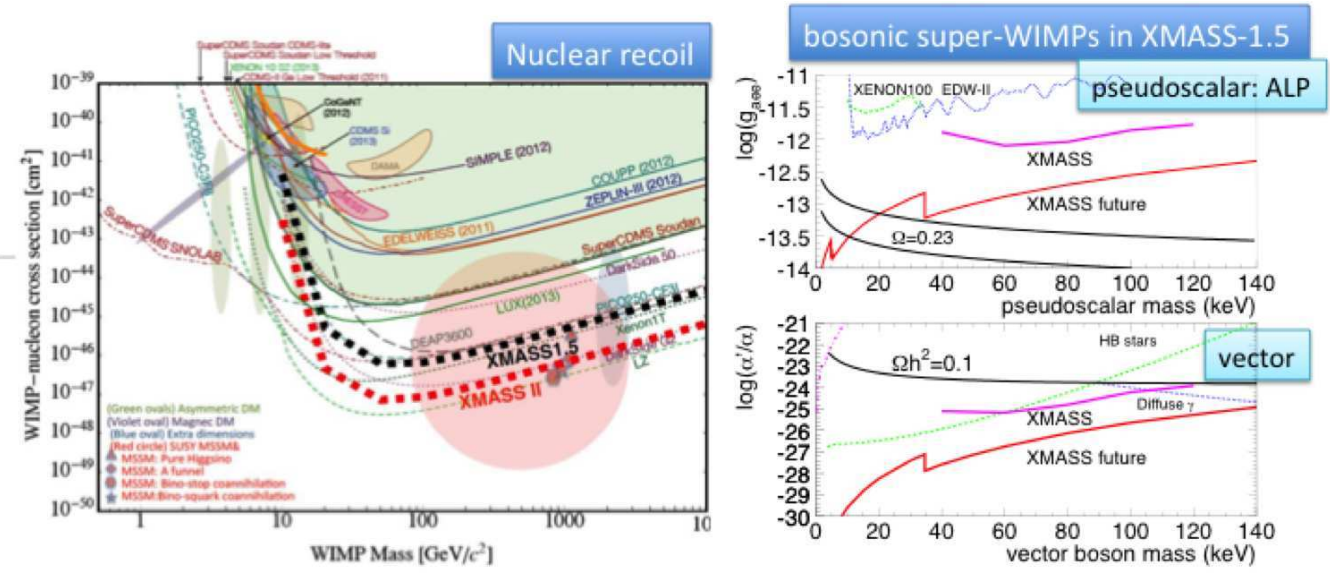

Figure 10: Estimated sensitivity of the XMASS-1.5 for WIMPs (left) and for superWIMPs (right). See text for details.

happening on the surface were not directly detected by PMTs close to the vertex. This caused the events sometimes to be unrecognizable as surface events and increased the background in the fiducial volume. However, PMTs with convex-shaped photocathodes (Fig. 7, right) are more efficient for detecting surface events. In fact, our simulation shows that three PMTs surrounding the event vertices detected $40 \%-50 \%$ of photoelectrons. With this improvement, we can fully exploit the self-shielding effect and reduce background down to the $10^{-5} \mathrm{~kg}^{-1} \mathrm{keV}^{-1}$ day ${ }^{-1}$, which is limited by the $p p$ solar neutrino signal. Figure 10 (left) shows the sensitivity of the XMASS-1.5 (5-ton total and 1-ton fiducial) and XMASS-II (25-ton total and 10-ton fiducial mass). With these future detectors, a sensitive search for WIMPs could be conducted. Further improvements would be done by discriminating nuclear recoil from $p p$ solar neutrino signals utilizing pulse-shape information. 
Figure 10 (right) shows the estimated sensitivity for the superWIMPs. Large improvements of background enable us to expand the mass range of the search and the sensitivity of the coupling of superWIMPs to electrons. It is also possible to explore the parameter space for non-thermal production of the vector superWIMPs.

\section{Summary}

The XMASS project aims to search for dark matter, low-energy solar neutrino signals, and neutrinoless double beta decay. The XMASS-I successfully provided various types of sensitive searches, including those for low-mass WIMPs, double-electron capture, inelastic scattering on ${ }^{129} \mathrm{Xe}$, and bosonic superWIMPs, and a dark matter search by annual modulation. To increase the sensitivity, we are planning to construct larger detectors with improved photosensors. With the expected background $10^{-5} \mathrm{~kg}^{-1} \mathrm{keV}^{-1} \mathrm{day}^{-1}$ caused by $p p$ solar neutrinos, a sensitivity better than $10^{-46} \mathrm{~cm}^{2}$ to the WIMP-nucleon cross section is expected. This enables us to search for WIMPs in the parameter region predicted by the supersymmetric theories. It is also highly sensitive to other candidates such as bosonic superWIMPs giving traces as electron-recoil events.

\section{References}

[1] K. Abe et al. (XMASS collaboration), Phys. Lett. B 719 (2013) 78.

[2] K. Abe et al. (XMASS collaboration), arXiv:1511.04807.

[3] R. Bernabei et al. Eur. Phys. J. C (2013) 73:2648.

[4] K. Abe et al. (XMASS collaboration), arXiv: 1510.00754.

[5] H. Uchida et al. (XMASS collaboration), Prog. Theor. Exp. Phys. 2014063 C01.

[6] K. Abe et al. (XMASS collaboration), Phys. Rev. Lett. 113 (2014) 121301. 